\title{
CIRUGÍA CORRECTORA DE LA INCURVACIÓN PENEANA
}

\author{
J.A. PORTILlO MARTÍN, M.A. CORREAS GÓMEZ, M.A. RADO VELÁSQUEZ, \\ J.L. GUTIÉRREZ BAÑOS, B. MARTÍN GARCÍA, R. HERNÁNDEZ RODRÍGUEZ, \\ J.I. DEL VALLE SCHAAN, A. ROCA EDREIRA, A. HERNÁNDEZ CASTRILLO, \\ F. RUIZ IZQUIERDO
}

\author{
Servicio de Urología. Hospital Universitario Valdecilla. Facultad de Medicina. \\ Universidad de Cantabria. Santander. \\ Actas Urol Esp. 27 (2): 97-102, 2003
}

\section{RESUMEN}

"CIRUGÍA CORRECTORA DE LA INCURVACIÓN PENEANA"

OBJETIVOS: Evaluar las técnicas de corporoplastia aplicadas a 59 pacientes afectos de incurvación peneana, tanto congénita como adquirida.

PACIENTES Y MÉTODO: Desde abril de 1991 hasta diciembre del 2001 se aplicaron 39 técnicas de Nesbit, 12 de Ruiz-Castañé y 8 de Essed-Schröeder en 44 pacientes con incurvación congénita y 15 con enfermedad de Peyronie (edad media de 25 años). En cinco casos se utilizó material reabsorbible y en el resto irreabsorbible, aplicando puntos invertidos para enterrar los nudos.

RESULTADOS: Con un seguimiento medio de 12 meses se ha obtenido la corrección de la incurvación en el $86 \%$ de los casos. Cuatro de los cinco casos en que se utilizó material reabsorbible requirieron segunda intervención por reincurvación. Un caso de acortamiento de pene, otro de hipoestesia glandar, un edema prolongado prepucial y cinco casos de palparse los puntos fueron las complicaciones más reseñables. No se observaron diferencias en cuanto a resultados y complicaciones según la técnica empleada.

CONCLUSIONES: Con las tres técnicas empleadas se ha obtenido un buen resultado cosmético y funcional, que coincide con las series publicadas en la literatura. Preferimos la sutura irreabsorbible para evitar el alto índice de reincurvaciones con el material absorbible.

PALABRAS CLAVE: Incurvación peneana. Corporoplastia. Complicaciones quirúrgicas.

\section{ABSTRACT}

\section{“CORRECTIVE SURGERY OF PENILE CURVATURE”}

OBJECTIVE: To evaluate the corporoplasty techniques performed on 59 patients to correct congenital penile curvature and Peyronie's disease.

PATIENTS AND METHOD: From april 1991 to december 2001, 39 Nesbit technique, 12 Ruiz-Castañé method and 8 Essed-Schröeder procedure were performed on forty-four congenital curvatures and fiftean Peyronie's diseases. The mean age was 24 years old. Trying to hide the knots, in all patients except five a nonabsorvable inverting sutures were placed.

RESULTS: The mean follow-up was 12 months. Postoperatively, 53 patients (86\%) had satisfactory cosmetic and functional results. Four patients of five with absorvable sutures had residual curvature which needed another successful surgical correction. One patient complained of penile shortening, one of glans hypoaesthesia, one of foreskin oedema and five of palpable plication sutures. No differences were found depending on the applied surgical technique.

CONCLUSIONS: The results reported are in accordance with the literature showing a higher recurrence rate with the absorvable sutures. The outcome is very similar with the three described techniques.

KEY WORDS: Penile curvature. Corporoplasty. Surgical complications. 
$\mathrm{L}$ a incurvación de pene tanto congénita como adquirida es una consulta frecuente en los servicios de urología. El hombre observa como el pene se incurva en erección, siendo ésta dolorosa y/o dificultando la penetración vaginal.

Es una enfermedad limitante de la actividad sexual, ya sea por alteración física del pene, como además por alteración psicológica, sobre todo en jóvenes.

A continuación pasamos a referir nuestra experiencia quirúrgica en aquellos casos que aceptaron la cirugía como tratamiento definitivo.

\section{PACIENTES Y MÉTODO}

Desde abril de 1991 hasta diciembre del 2001, hemos intervenido quirúrgicamente en nuestro Hospital Universitario Valdecilla de Santander a 59 pacientes, en edades comprendidas entre los 17 y los 66 años (edad media de 25).

La sintomatología era básicamente la incurvación en 50 casos $(84,7 \%)$, refiriendo además otros 7 pacientes dolor eréctil o coital y dos disfunción eréctil.

La etiología de la incurvación la consideramos en 44 casos $(74,5 \%)$ congénita y en 15 adquirida. En 9 de éstos se palpaba placa de Peyronie y uno de ellos había sido operado de Dupuytren.

La incurvación ventral la observamos en 26 casos (44\%), dorsal en 11 (18,6\%), lateral izda en 19 (32,4\%), y lateral dcha en 3 (5\%). En 34 casos $(57,2 \%)$ era de unos $30^{\circ}$ y el resto entre 30 y $60^{\circ}$.

En los 44 casos congénitos referían tener la incurvación "de siempre" y el resto llevaba una evolución al menos de más de un año. En 6 casos se había aplicado algún tipo de tratamiento local (mayoritariamente infiltración local sobre la placa con ergoteína) sin resultado efectivo.

El método diagnóstico más utilizado ha sido la autofotografía en 24 casos (40,6\%), teniendo que recurrir en otros 15 casos $(25,4 \%)$ al test con PGE 1 intracavernosa. En los 20 casos restantes se practicó este último test en el quirófano.

Desde hace unos 3-4 años deben firmar consentimiento informado en donde se les indica específicamente la posibilidad de acortamiento del pene y de la palpación de los puntos, entre otras menores complicaciones.

De los 59 casos, 36 fueron operados con anestesia general, 11 con raquídea y 12 con local + sedación.
De forma profiláctica se administran tres dosis de amoxicilina-clavulánico para evitar infecciones, aunque en general se trata de una cirugía limpia.

En 4 casos se colocó sonda uretral durante la cirugía.

El tipo de incisión fue: en 39 casos circuncisión, en 17 longitudinal sobre el cuerpo peneano y en 3 subcoronal sin circuncisión.

En 41 casos fue necesario realizar 1 ó 2 incisiones sobre la albugínea, en 17 se practicaron 3 ó 4 , y en un caso 6 incisiones.

En 39 pacientes se aplicó la técnica de Nesbit, resecando elípticamente un parche de albugínea, en 12 casos se delineó la elipsis pero sin resecar la pastilla sino enterrándola (Técnica de RuizCastañé), y en 8 casos se procedió a la plicatura de la albugínea sin incidirla (Técnica de EssedSchröeder).

En los primeros 5 casos se realizó la sutura con material reabsorbible y el resto con no reabsorbible, normalmente prolene de 3-4 ceros. Los puntos fueron invertidos para que los nudos quedaran enterrados por dentro de la albugínea.

No se dejó sonda post-operatoria en ningún caso y la mayoría de los pacientes fueron dados de alta al día siguiente de la cirugía.

El seguimiento medio tras la cirugía en esta serie es de 12 meses.

\section{RESULTADOS}

En 56 casos no observamos ninguna complicación precoz. En 2 casos hubo infección local de la herida y en un caso hematoma peneano que se resolvió espontáneamente.

Como complicaciones tardías, la más importante fue la reincurvación en 6 casos correspondiendo 2 de ellas a las incurvaciones adquiridas. Cuatro de ellos fueron reoperados de forma satisfactoria. Es importante reseñar que de los 5 pacientes en que se utilizó material reaborbible, 4 reincurvaron con el paso del tiempo. De los 6 casos recidivados, uno es de la técnica de Essed, cuatro de Nesbit y uno de Ruiz-Castañé.

Cinco pacientes se quejaron de molestia local de los puntos que fue cediendo con el paso del tiempo.

Un paciente no circuncidado tuvo edema prolongado del prepucio que fue desapareciendo gradualmente a lo largo de un año. 
Y otro paciente muy particular se quejó de acortamiento de pene, además de molestias locales debido a los puntos. A pesar de haberse corregido la incurvación, insistió para que le extrajéramos los puntos. Ante nuestra resistencia inicial, se fue a otro centro donde se los quitaron a los 6 meses de la cirugía, no volviendo a consulta y desconociendo por tanto su evolución.

Desde el punto de vista del paciente, 51 (86\%) de ellos (incluidos los 4 reoperados) estaban satisfechos con el resultado. Otros 2 reconocían una mejoría del $50 \%$ y 3 decían no haber mejorado lo suficiente. Tres pacientes no volvieron a revisión.

Como tratamiento complementario, además de los 4 reoperados, 2 pacientes con Peyronie están en tratamiento con Sildenafilo 50 mgrs por cierto grado de disfunción eréctil (DE) que ya presentaban previamente.

\section{DISCUSIÓN}

Desde que Nesbit ${ }^{1}$ en 1965 describiera su técnica para corregir la incurvación congénita de pene, la mayoría de los urólogos hemos seguido sus principios para tratar esta patología, no infrecuente en nuestras consultas.

Asímismo, se ha aplicado la misma técnica en las incurvaciones adquiridas (enfermedad de Peyronie, postraumáticas, post-quirúrgicas), sobre todo en el Peyronie, ya que los tratamientos médicos utilizados no logran en general resultados positivos. Se ha empleado la técnica de incisión de la placa y parche con vena safena sobre todo cuando la incurvación y dureza de la placa son importantes ${ }^{2,3}$, pero en casos menos acusados la técnica de Nesbit también tiene su papel.

La etiología de la incurvación congénita no se sabe con exactitud. Se cree que se debe a un fallo del desarrollo embrionario. Al principio el pene es curvo ventral y poco a poco se va enderezando hasta conseguir el estado recto final ${ }^{4}$. Un déficit androgénico o la insensibilidad a los andrógenos puede producir detención en el desarrollo con curvatura, asociada o no a hipoplasia uretral y/o afectación del cuerpo esponjoso, fascia de Buck y dartos. También puede haber una desproporción de los cuerpos cavernosos lateralizando el pene hacia el cuerpo más desarrollado.
Según la clasificación de Devine-Horton de 1973, el chordée sin hipospadias puede ser de tres tipos:

I. Uretra justo debajo de la piel con ausencia de esponjosa, f. de Buck y dartos.

II. Uretra y esponjosa normales, con anormalidad de la f. de Buck y del dartos.

III. Uretra, esponjosa y f. de Buck normales y alteración del dartos.

En los tipos II y III se pueden aplicar técnicas de plicatura, pero en el tipo I habrá además que tratar la uretra distal como un hipospadias.

La capa albugínea está compuesta por colágeno y elastina, siendo su distribución en capas, la externa longitudinal y la interna circular. En estudio ultraestructural realizado sobre la albugínea del lado de máxima curvatura se observa una distribución caótica de las fibras de colágeno, formando apelotonamientos. Las fibras están fragmentadas con desaparición de las estriaciones normales y transformación en tejido fibroso y granuloso denso ${ }^{5}$.

La incurvación congénita suele ser ventral (50\%) o bien con algún grado de lateralidad (45\%). Sólo en un 5\% suele ser dorsal sobre todo en penes largos $^{6}$, situación contraria de la incurvación adquirida, en la que la dorsal es la más frecuente.

El diagnóstico se realiza por medio de la autofotografía (técnica de Kelami) o bien provocando la erección con estímulo visual y/o PGE1. Otros autores realizan estudios exhaustivos con Rigiscan y Eco-doppler sobre todo en la enfermedad de Peyronie ${ }^{7}$.

Las técnicas quirúrgicas empleadas para corregir la incurvación tienen la finalidad de acortar la albugínea en la zona de máxima convexidad.

La técnica original de Nesbit consiste en resecar una pastilla transversal de albugínea y cerrar el defecto con puntos sueltos, realizando tantas incisiones como sean necesarias para corregir la incurvación. En 1985 Kelami añadió el detalle técnico de coger un pellizco de albugínea con una pinza de Allis para observar mejor la corrección y sobre ella incidirla. A partir de entonces algunos llamaron a esta técnica de Nesbit-Kelami ${ }^{8}$.

En casos excepcionales en que además de incurvación se observa rotación peneana, se propone la extirpación de elipses albugíneas para de esta forma corregir la curvatura y rectificar la torsión ${ }^{9,10}$. 
En 1993 Ruiz-Castañé en nuestro país propone el no resecar la albuginea tras incidirla, sino que proceder a enterrarla al aproximar los bordes incididos. Sugiere que de esta forma se disminuyen las complicaciones hemorrágicas. Otra modificación del Hospital Miguel Servet consiste en incidir la albuginea pero sin perforarla, aproximando los bordes con un punto central irreabsorbible y otros dos laterales con reabsorbible ${ }^{11}$.

Otro tipo de técnicas son las de plicatura. Según refiere Baskin ${ }^{12}$ parece que la primera técnica fue descrita por Philip Syng Physi en el siglo XIX, quien la utilizó para corregir un chordée sin hipospadias. Sin embargo fueron Essed y Schröeder quienes popularizaron la plicatura en $1985^{13}$, aunque curiosamente casi de forma simultánea Ebbehoj y Metz publicaron la misma técnica ${ }^{14}$. Consiste en plicar la albugínea sin incidirla, invirtiendo el sentido del punto para que el nudo quede enterrado.

Serrate ${ }^{15}$ describió su técnica en 1989 consistente en, tras aplicar el punto de plicatura, practicar varias incisiones longitudinales y transversales no perforantes de la albugínea que queda en medio. De esta forma cree favorecer la pérdida de consistencia de la albugínea y estimular su cicatrización.

Un tipo diferente de técnica es la que preconizó Saalfeld en 1973 basándose en el principio de la piloroplastia de Heincke-Mikulicz. Consiste en incidir la albugínea de forma longitudinal y cerrándola en sentido transversal. Yachia en 1990 popularizó la técnica y con este nombre se conoce hoy en día ${ }^{16}$.

Por último habría que describir la técnica descrita por Perovic ${ }^{17}$. Separa todos los elementos peneanos, individualizando la uretra con el glande por un lado, los cuerpos cavernosos por otro y finalmente el paquete vásculo-nervioso. Procede a la plicatura de los c. cavernosos donde sea necesario y así consigue que nunca tenga acortamientos de pene. Bien al contrario, puede utilizar esta técnica para intercalar algún tipo de tejido entre los c. cavernosos y el glande (por ejemplo cartílago costal) y así conseguir alargamiento de pene.

La anestesia admite todas las variantes, incluso la local, pero la mayoría prefiere al menos la regional.
El abordaje del pene suele ser subcoronal, con circuncisión asociada o no, o bien longitudinal sobre la zona en donde se van a aplicar los puntos. Casi todos los autores consultados provocan la erección con suero fisiológico (técnica de GittesMcLaughin descrita en 1974) y a partir de ese momento practicar la técnica que se crea conveniente.

Independientemente de la técnica que se practique, la tendencia es a invertir los puntos para enterrar todo lo que se pueda los puntos y evitar el que se palpen o la formación de granulomas exuberantes.

Aunque a veces se usa sonda de Foley intraoperatoria, no parece que sea necesario dejar la sonda 24-48 horas como hacen algunos autores $^{18,19}$.

Es muy difícil comparar los resultados de las distintas técnicas, e incluso de una misma técnica ya que se suelen mezclar las patologías (congénitas y adquiridas), en unos casos se utiliza material irreabsorbible y en otros absorbible y a veces el seguimiento es corto.

En general los éxitos suelen estar por encima del 80\%, entendiendo por tal la desaparición de la incurvación, a veces incluso tras dos operaciones (Tablas I y II). Los mejores resultados se obtienen en las curvaturas congénitas, siendo peores en el Peyronie $^{20-23}$. Incluso pacientes de éstos con disfunción eréctil previa la siguen teniendo tras la corrección de la incurvación. Chanal ${ }^{20}$ refiere sólo un buen resultado en el 29\% de Peyronie, ya que el $57 \%$ queda con incurvación residual y otro $14 \%$ con incurvación severa.

La complicación más temida a medio plazo es el acortamiento del pene ${ }^{7,18,20-22,24,25}$, así como el abultamiento que producen las suturas ${ }^{20,21,25,26}$. Otra complicación menos frecuente es la hipoestesia del glande que se puede producir si nos acercamos mucho al paquete vásculo-nervioso ${ }^{18,20,22}$.

Se calcula que se produce un acortamiento del pene de 1-2 mm por cada 10 o de incurvación, siendo más frecuente en el Peyronie que en las congénitas $^{8}$.

Nosotros sólo hemos podido constatar un caso claro de queja de acortamiento peneano, cinco se palpaban los puntos y una hipoestesia que desapareció con el tiempo. 
TABLA I

COMPARACIÓN DE SERIES

\begin{tabular}{|c|c|c|c|c|c|}
\hline Autor & № casos & Tipo & Técnica & Sutura & Éxito \\
\hline Nooter, 1994 & 55 & $\begin{array}{l}33 \text { Peyronie } \\
22 \text { Congénito }\end{array}$ & Essed & No absorbible & $83 \%$ \\
\hline Sassine, 1994 & 55 & $\begin{array}{l}23 \text { Peyronie } \\
32 \text { Congénito }\end{array}$ & Yachia & Absorbible & $94 \%$ \\
\hline Poulsen, 1995 & 175 & $\begin{array}{l}57 \text { Peyronie } \\
118 \text { Congénito }\end{array}$ & $\begin{array}{l}143 \text { Nesbit } \\
32 \text { Essed }\end{array}$ & Absorbible & $\begin{array}{l}87 \% \\
60 \%\end{array}$ \\
\hline López-M., 1995 & 21 & Congénito & Nesbit & Absorbible & $100 \%$ \\
\hline Thiounn, 1998 & 60 & $\begin{array}{c}29 \text { Peyronie } \\
25 \text { Congénito } \\
6 \text { Postr. }\end{array}$ & Essed & No absorbible & $87 \%$ \\
\hline Padilla, 1998 & 22 & $\begin{array}{l}11 \text { Peyronie } \\
11 \text { Congénito }\end{array}$ & Essed & No absorbible & $73 \%$ \\
\hline Daitch, 1999 & 28 & $\begin{array}{l}19 \text { Peyronie } \\
9 \text { Congénito }\end{array}$ & Yachia & Absorbible & $89 \%$ \\
\hline
\end{tabular}

TABLA II

COMPARACIÓN DE SERIES

\begin{tabular}{|c|c|c|c|c|c|}
\hline Autor & № casos & Tipo & Técnica & Sutura & Éxito \\
\hline Popken, 1999 & 105 & Congénito & Nesbit & Absorbible & $95 \%$ \\
\hline Schultheiss, 2000 & 61 & $\begin{array}{l}21 \text { Peyronie } \\
40 \text { Congénito }\end{array}$ & Essed & No absorbible & $70,5 \%$ \\
\hline Friedrich, 2000 & 40 & $\begin{array}{l}11 \text { Peyronie } \\
19 \text { Congénito }\end{array}$ & Essed & No absorbible & $81 \%$ \\
\hline Chanal, 2001 & 44 & Peyronie & Essed & No absorbible & $29 \%$ \\
\hline Rodríguez, 2001 & 44 & $\begin{array}{l}17 \text { Peyronie } \\
27 \text { Congénito }\end{array}$ & Propia & Mixta & $100 \%$ \\
\hline Valdecilla, 2002 & 59 & $\begin{array}{l}15 \text { Peyronie } \\
44 \text { Congénito }\end{array}$ & $\begin{array}{c}39 \text { Nesbit } \\
12 \text { Ruiz-Cas. } \\
8 \text { Essed }\end{array}$ & $\begin{array}{c}5 \text { Absorbible } \\
54 \text { No absorbible }\end{array}$ & $86 \%$ \\
\hline
\end{tabular}

La tasa global de buenos resultados del $86 \%$ de nuestra serie entra dentro de lo esperado. A raíz de nuestra mala experiencia con el material absorbible, aplicamos siempre irreabsorbible (prolene 3/0).

No existen estudios randomizados que verifiquen qué técnica es mejor ni qué material es más fiable, por tanto de momento lo lógico es aplicar aquella con la que cada uno obtenga mejores resultados.

\section{REFERENCIAS}

1. NESBIT RM.: Congenital curvature of the fallus: report of three cases with description of corrective operation. J Urol 1965; 93: 230-233.

2. MONTORSI F, SALONIA A, MAGA T et al.: Evidence based assessment of long-term results of plaque incision and vein grafting for Peyronie's disease. $J$ Urol 2000; 163: 1.704-1.708.

3. YURKANIN JP, DEAN R, WESSELLS H.: Effect of incision and saphenous vein grafting for Peyronie's disease on penile length and sexual satisfaction. $J$ Urol 2001; 166: 1.769-1.773. 
4. DASKALOPOULOS EI, BASKIN L, DUCKETT JW, SNYDER HM.: Congenital penile curvature (chordee without hypospadias). Urology 1993; 42 (6): 708712 .

5. DAREWICZ B, KUDELSKI J, SZYNAKA B, NOWAK HF, DAREWICZ J.: Ultrasestructure of the tunica albuginea in congenital penile curvature. $J$ Urol 2001; 166: 1.766-1.768.

6. ADAMS MC, CHALIAN VS, RINK RC.: Congenital dorsal penile curvature: a potencial problem of the long phallus. J Urol 1999; 161: 1.304-1.307.

7. DAITCH JA, ANGERMEIER KW, MONTAGUE DK.: Modified corporoplasty for penile curvature: longterm results and patient satisfaction. J Urol 1999; 162: 2.006-2.009.

8. POPKEN G, WETTERAUER U, SCHUTZE-SEEMANN W, DECKART AB, SOMMERKAMP H.: A modified corporoplasty for treating congenital penile curvature and reducing the incidence of palpable indurations. Br J Urol Int 1999; 83: 71-75.

9. BELGRANO E, LIGUORI G, TROMBETTA C, SIRACUSANO S.: Correction of complex penilee deformities by modified Nesbit procedure asymmetric tunica albuginea excision. Eur Urol 1999; 38: 172-176.

10. SLAWIN KM, NAGLER HM.: Treatment of congenital penile curvature with penile torsion: a new twist. $J$ Urol 1992; 147: 152-154.

11. RODRÍGUEZ L, CHANTADA V, FITER L, MONCADA I, CRUZ N, MARTÍN A.: Incurvación congénita del pene. Tema monográfico del LXVI Congreso Nacional de la AEU titulado "Enfermedad de Peyronie y otras alteraciones morfométricas del pene”. Celebrado en Granada en Mayo 2001. Págs 101-119.

12. BASKIN LS, DUCKETT JW, LUE TF.: Penile curvature. Urology 1996; 48 (3): 347-356.

13. ESSED E, SCHROEDER FH.: New surgical treatment of Peyronie disease. Urology 1985; 25: 582587.

14. EBBEHOJ J, METZ P.: New operation for krummerick (penile curvature). Urology 1985; 26: 76-78.

15. SERRATE RG, RIUS G, REGUE R, PRATS J.: Modificación técnica en el tratamiento quirúrgico de la curvatura de pene. Arch Esp Urol 1989; 42 (4): 313-315.

16. YACHIA D.: Modified corporoplasty for the treatment of penile curvature. J Urol 1990; 143: 80-82.
17. PEROVIC SV, DJORDJEVIC MLJ, DJAKOVIC NG.: A new approach to the treatment of penile curvature. J Urol 1998; 160: 1.123-1.127.

18. THIOUNN N, MISSIRLIU A, ZERBIB M et al.: Corporeal plication for surgical correction of penile curvature. Eur Urol 1998; 33: 401-404.

19. LÓPEZ JJ, FERNÁNDEZ DEL BUSTO E, CORTIÑAS R, EGEA J, RIVERO MD.: Cavernoplastia de Nesbit en el tratamiento de la incurvación congénita de pene. Nuestra casuística. Arch Esp Urol 1995; 48 (3): 294-297.

20. CHANAL R, GOGOL NK, SUNDARAM SK, WESTON PMT.: Corporal plication for penile curvature caused by Peyronie's disease: the patients' perspective. $\mathrm{Br}$ $J$ Urol Int 2001; 87: 352-356.

21. FRIEDRICH MG, EVANS D, NOLDUS J, HULAND H.: The correction of penile curvature with the EssedSchröder technique: a long-term follow-up assessing functional aspects and quality of life. $\mathrm{Br} J$ Urol Int 2000; 86: 1.034-1.038.

22. NOOTER RI, BOSCH JLHR, SCHRÖDER FHS.: Peyronie's disease and congenital penile curvature: long-term results of operative treatment with the plication procedure. Br J Urol 1994; 74: 497-500.

23. POULSEN J, KIRKEBY HJ.: Treatment of penile curvature; a retrospective study of 175 patients operated with plication of the tunica albuginea or with the Nesbit procedure. Br J Urol 1995; 75: 370-374.

24. PADILLA J, MARIN JC, LEKUMBERRI D, ALBISU A, ACHA M, PERTUSA C.: Plicatura de la albugínea como tratamiento para la incurvación de pene. Act Esp Urol 1998; 22 (10): 853-856.

25. SCHULTHEISS D, MESCHI MR, HAGEMANN J, TRUSS MC, STIEF CG, JONAS U.: Congenital and acquired penile deviation treated with the Essed plication method. Eur Urol 2000; 38: 167-171.

26. SASSINE AM, WESPES E, SCHULMAN CC.: Modified corporoplasty for penile curvature: 10 years` experience. Urology 1994; 44 (3): 419-421.

Dr. J.A. Portillo Martín

Avda. del Faro, 1 - B. 2, $3^{\circ}$ B

39012 Santander (Cantabria)

(Trabajo recibido el 1 abril de 2002) 